\title{
Hyperhomocysteinemia and Increased Oxidative Stress Levels Are Associated with Impaired Membrane Fluidity of Red Blood Cells in Hypertensive and Normotensive Men: An Electron Spin Resonance Investigation
}

\author{
Kazushi Tsuda \\ ${ }^{1}$ Cardiovascular and Metabolic Research Center, Kansai University of Health Sciences, Osaka, Japan; ${ }^{2}$ Division of Cardiology, \\ School of Medicine, Wakayama Medical University, Wakayama, Japan. \\ Email: tsudak@mail.wakayama-med.ac.jp
}

Received November $30^{\text {th }}, 2012$; revised December $30^{\text {th }}, 2012$; accepted January $12^{\text {th }}, 2013$

\begin{abstract}
Hyperhomocysteinemia and oxidative stress may be strongly linked to hypertension, atherosclerosis and other cardiovascular diseases. The present study was performed to investigate possible relationships among plasma total homocysteine, plasma 8-iso-prostaglandin F2 $\alpha$ (8-isoPG F2 $\alpha$ : an index of oxidative stress), and membrane fluidity (a reciprocal value of membrane microviscosity) in hypertension. We measured the membrane fluidity of red blood cells (RBCs) in hypertensive and normotensive men using an electron spin resonance (ESR) and spin-labeling method. Membrane fluidity of RBCs was significantly decreased in hypertensive men compared with normotensive men. Plasma total homocysteine levels were significantly higher in hypertensive men than in normotensive men, and correlated with plasma 8 -isoPG F2 $\alpha$. In contrast, plasma nitric oxide (NO)-metabolites (an index of endothelial function) were lower in hypertensive men than in normotensive men. The reduced membrane fluidity of RBCs was associated with increased total homocysteine and plasma 8-isoPG F2 $\alpha$ levels and decreased plasma NO-metabolite levels. Multivariate regression analysis showed that, after adjusting for general risk factors, plasma total homocysteine and 8-isoPG F2 $\alpha$ were significant determinants of membrane fluidity of RBCs, respectively. These results suggest that hyperhomocysteinemia and oxidative stress with endothelial dysfunction might have a close correlation with impaired rheologic behavior of RBCs and circulatory disorders in hypertensive men.
\end{abstract}

Keywords: Homocysteine; Oxidative Stress; 8-Iso-Prostaglandin F2 $\alpha$; Nitric Oxide; Membrane Fluidity; Red Blood Cell; Electron Spin Resonance; Hypertension

\section{Introduction}

Evidence indicates that many cardiometabolic factors, including hyperhomocysteinemia, might have a pivotal role in the progression of hypertension, atherosclerosis and other cardiovascular diseases [1-3]. Wald et al. [2] demonstrated the strong link between homocysteine and cardiovascular diseases, and proposed that lowering plasma homocysteine concentration would reduce the risk of ischemic heart disease, deep vein thrombosis, and stroke. It has also been shown that endothelium-dependent vasodilation was impaired in humans with elevated plasma homocysteine [4], suggesting that hyperhomocysteinemia could reduce the nitric oxide (NO)-availability and actively participate in the pathogenesis of vascular dysfunction.

On the other hand, it is well recognized that oxidative stress might be associated with increased risk of vascular damage, cardiovascular diseases and the metabolic syndrome [5-9]. Recently, it has been shown that plasma 8-iso-prostaglandin F2 $\alpha$ (8-iso-PG F2 $\alpha$ ) may be a reliable index of oxidative stress in humans [10-14]. It was demonstrated that plasma concentration of 8-iso-PG F2 $\alpha$ was significantly increased in subjects with essential hypertension compared with normotensive subjects $[10,11,14]$. It was also shown that plasma 8-iso-PG F2 $\alpha$ levels were elevated in patients with coronary artery disease, especially in those with hypertension $[12,13]$.

Abnormalities in physical properties of the cell membranes may underlie the defects that are strongly linked to hypertension $[15,16]$. An electron spin resonance (ESR) and spin-labeling method has been developed to evaluate the membrane fluidity (a reciprocal value of membrane microviscosity) and perturbations of the membrane function by external agents $[15,16]$. Using the ESR method, 
we have been performing a series of experiments regarding the membrane fluidity in subjects with essential hypertension [14,17-23]. The results suggest that the membrane fluidity of red blood cells (RBCs) was significantly lower in hypertensive subjects than in normotensive subjects, indicating that the cell membranes were stiffer and less fluid in essential hypertension [14,17-23]. Because the deformability of RBCs may be highly dependent on the membrane fluidity, the reduction in membrane fluidity of RBCs could cause a disturbance in the blood rheologic behavior and in the microcirculation, which might contribute to the pathophysiology of hypertension and other circulatory disorders $[15,16]$. In the studies presented previously, we showed that hyperhomocysteinemia and oxidative stress might be associated with reduced membrane fluidity of RBCs in hypertensive subjects, respectively $[14,17,22,23]$, although interactions between hyperhomocysteinemia and oxidative stress and their role in the regulation of membrane fluidity of RBCs remain unclear. In the present study, therefore, we investtigated possible relationships among plasma total homocysteine levels, oxidative stress and membrane fluidity of RBCs in hypertensive and normotensive men using the ESR method.

\section{Subjects and Methods}

\subsection{Subjects}

A total of 32 men with untreated essential hypertension (systolic blood pressure more than $140 \mathrm{mmHg}$ and/or diastolic blood pressure more than $90 \mathrm{mmHg}$ ) were studied and compared with 21 age-matched normotensive men. The characteristics and laboratory findings in both groups were shown in Table 1. Subjects who had a history of other diseases such as hematologic or hepatic disorders were excluded. All men were non-smokers. They had similar life styles and dietary habits, and were instructed to avoid any changes in dietary habits before the study. The study was approved by a local research committee of Kansai University of Health Sciences. Informed consent was obtained from all participants after they were informed about the nature and objective of the study.

\subsection{Electron Spin Resonance Measurements of Red Blood Cells}

Blood sampling was performed by venipuncture after a 30 minutes of bed rest while fasting. The procedures of RBC preparation and ESR measurements were shown previously [17-23]. We used heparin as the anti-coagulant (10 U heparin/10 $\mathrm{ml}$ blood). The plasma and buffy coat were carefully removed by centrifugation at $155 \mathrm{~g}$ for $10 \mathrm{~min}$ at $4^{\circ} \mathrm{C}$. Then, the sedimented RBCs were re- suspended in the isotonic buffer $(140 \mathrm{mmol} / \mathrm{L} \mathrm{NaCl} 20$ $\mathrm{mmol} / \mathrm{L}$ Tris-HCL, $\mathrm{pH}$ 7.4). The RBC solution was centrifuged at $155 \mathrm{~g}$ for $10 \mathrm{~min}$ at $4^{\circ} \mathrm{C}$. This procedure was repeated three times and the washed RBCs were suspended in the isotonic buffer $(140 \mathrm{mmol} / \mathrm{L} \mathrm{NaCl}$ and 20 $\mathrm{mmol} / \mathrm{L}$ Tris-HCl buffer, $\mathrm{pH} 7.4$ ) at a hematocrit of $50 \%$. The RBC suspension $(300 \mu \mathrm{l})$ was incubated with the solution $(100 \mu \mathrm{l})$ containing fatty acid spin label agent (5-nitroxide stearate $5 \times 10^{-5} \mathrm{~mol} / \mathrm{L}$, Aldrich Co. Ltd., Milwaukee, Wisconsin, USA) for 2 hours with gentle shaking at $37^{\circ} \mathrm{C}$ [17-19]. After incubation, the ESR measurements were immediately performed using an ESR spectrometer (model Jeol JES-FE2XG, Nihon Denshi, Tokyo, Japan) with a microwave unit (model Jeol ESSCXA, Nihon Denshi) [17-23]. The microwave power was $5 \mathrm{~mW}$ and the modulation frequency was $100 \mathrm{KHz}$ with a modulation amplitude of $0.2 \mathrm{~m}$ tesla (T). The temperature of the measurement was controlled at $30^{\circ} \mathrm{C}$. For indices of membrane fluidity, we evaluated the values of outer and inner hyperfine splitting (2T' // and 2T' $\perp$ in tesla (T), respectively) in each ESR spectrum (Figure 1) and calculated the order parameter (S) [20]. The greater the value of the order parameter (S) was, the lower the membrane fluidity of RBCs was [17-23].

Table 1. Clinical characteristics and laboratory findings of hypertensive (HT) and normotensive (NT) men.

\begin{tabular}{|c|c|c|}
\hline & NT & HT \\
\hline Number of subjects & 21 & 32 \\
\hline Age (y.o.) & $64 \pm 3$ & $62 \pm 2$ \\
\hline Body mass index $\left(\mathrm{kg} / \mathrm{m}^{2}\right)$ & $24.1 \pm 0.6$ & $24.1 \pm 0.5$ \\
\hline Systolic blood pressure (mmHg) & $125 \pm 2$ & $146 \pm 1^{*}$ \\
\hline Diastolic blood pressure (mmHg) & $70 \pm 2$ & $86 \pm 1^{*}$ \\
\hline Heart rate (beats/min) & $76 \pm 1$ & $73 \pm 1$ \\
\hline Erythrocyte counts $\left(10^{4}\right.$ cells $\left./ \mu \mathrm{L}\right)$ & $452 \pm 10$ & $480 \pm 7$ \\
\hline Hemoglobin (g/dL) & $14.1 \pm 0.3$ & $14.2 \pm 0.2$ \\
\hline Hematocrit (\%) & $43.1 \pm 1.0$ & $43.0 \pm 1.0$ \\
\hline Leucocyte counts $\left(10^{3}\right.$ cells $\left./ \mu \mathrm{L}\right)$ & $5.5 \pm 0.2$ & $5.3 \pm 0.2$ \\
\hline Platelets $\left(10^{4}\right.$ cells $\left./ \mu \mathrm{L}\right)$ & $20 \pm 1$ & $22 \pm 1$ \\
\hline Total cholesterol (mg/dL) & $200 \pm 6$ & $213 \pm 6$ \\
\hline $\begin{array}{l}\text { High density lipoprotein cholesterol } \\
\qquad(\mathrm{mg} / \mathrm{dL})\end{array}$ & $\mathbf{5 0} \pm \mathbf{3}$ & $53 \pm 3$ \\
\hline $\begin{array}{l}\text { Low density lipoprotein cholesterol } \\
\text { (mg/dL) }\end{array}$ & $123 \pm 6$ & $126 \pm 5$ \\
\hline Triglycerides (mg/dL) & $137 \pm 17$ & $160 \pm 18$ \\
\hline Serum sodium $(\mathbf{m m o l} / \mathrm{L})$ & $140.5 \pm 0.4$ & $140.0 \pm 0.3$ \\
\hline Serum potassium $(\mathrm{mmol} / \mathrm{L})$ & $4.0 \pm 0.1$ & $4.0 \pm 0.1$ \\
\hline Serum creatinine $(\mathrm{mg} / \mathrm{dL})$ & $0.8 \pm 0.1$ & $0.9 \pm 0.1$ \\
\hline Fasting plasma glucose $(\mathrm{mg} / \mathrm{dL})$ & $113 \pm 6$ & $124+8$ \\
\hline
\end{tabular}

Values are means \pm SEM. $* \mathrm{P}<0.01$ between $\mathrm{HT}$ and NT. 


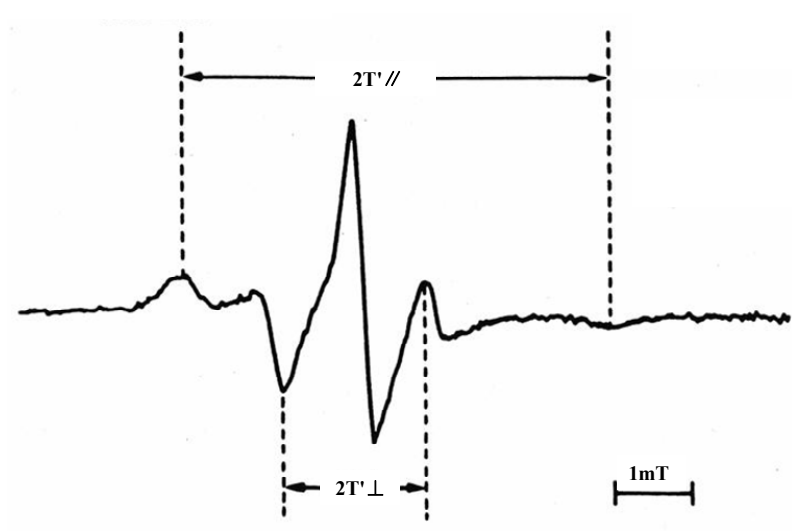

Figure 1. Representative electron spin resonance (ESR) spectrum of red blood cells (RBCs) for the fatty acid spinlabel agent (5-nitroxide stearate). $2 T^{\prime} / /$ : outer hyperfine splitting, 2T' $\perp$ : inner hyperfine splitting, $T$ : tesla We calculated the order parameter (S) from $2 T^{\prime} / /$ and $2 T^{\prime} \perp$ values according to the formula (20). $S=\left(a_{n} / a_{n}^{\prime}\right)\left(T^{\prime} / /-T^{\prime}\right.$ $\perp) /(\mathbf{T z z}-\mathbf{T x x}) \mathbf{a}_{\mathbf{n}} / \mathbf{a}_{\mathbf{n}}$ : isotropic coupling constant, Tzz and Txx: hyperfine constants. The greater the value of the order parameter (S) was, the lower the membrane fluidity of RBCs was (17 - 23).

\subsection{Analaysis of Plasma 8-Iso-Prostaglandin F2 $\alpha$ and Plasma Nitric Oxide-Metabolites (Nitrite and Nitrate)}

The levels of plasma 8-iso-PG F2 $\alpha$ (an index of oxidative stress) were determined by using an enzyme immunoassay (Cayman Chemicals Co., Ann Arbor, Michigan, USA) [11-13].

The plasma levels of NO-metabolites (nitrite and nitrate) (an index of endothelial function) were measured with an automated NO detector/high-performance liquid chromatography system (ENO 10, Eicom Co., Tokyo, Japan), as previously described [21]. Briefly, nitrite and nitrate in plasma were separated by a reverse-phase separation column, and the nitrate was reduced to nitrite in a reduction column. Nitrite was mixed with Griess reagents (sulfanilamide and naphthalene-ethylene diamine dihydrochloride), and the absorbance at $540 \mathrm{~nm}$ was measured by a flow-through spectrometer.

\subsection{Statistical Analysis}

Values are expressed as mean \pm SEM. The differences between hypertensive and normotensive men were analyzed using an unpaired Student's t-test. Linear regression analysis was performed to assess the relationships among membrane fluidity (order parameter; S) of RBCs, plasma total homocysteine, plasma 8-iso-PG F2 $\alpha$, and NO-metabolite levels. Multivariate regression analysis with membrane fluidity (order parameter; S) of RBCs as a dependent variable, and general risk factors (age, body mass index: BMI, plasma total cholesterol, fasting plasma glucose, and systolic blood pressure) and plasma total homocysteine, or plasma 8-iso-PG F2 $\alpha$ as independent variables was also performed. A P-value less than 0.05 was accepted as the level of significance.

\section{Results}

\subsection{Membrane Fluidity of Red Blood Cells in Hypertensive and Normotensive Men}

The order parameter (S) for the spin-label agents (5-nitroxide stearate) of RBC membranes in the ESR spectra was significantly higher in hypertensive (HT) men than in normotensive (NT) men (HT: $0.728 \pm 0.002, \mathrm{n}=32$, NT: $0.718 \pm 0.001, \mathrm{n}=21, \mathrm{P}<0.001)$. The finding indicated that membrane fluidity was decreased in hypertensive men compared with normoten- sive men.

\subsection{Plasma Total Homocysteine, Plasma 8-Iso-Prostaglandin F2 $\alpha$, and Plasma Nitric Oxide-Metabolite Levels in Hypertensive and Normotensive Men}

The plasma total homocysteine levels were significantly elevated in hypertensive men compared with normotensive men (HT: $12.7 \pm 0.8 \mu \mathrm{mol} / \mathrm{L}, \mathrm{n}=32$, NT: $10.2 \pm 0.4$ $\mu \mathrm{mol} / \mathrm{L}, \mathrm{n}=21, \mathrm{P}<0.05)$. The plasma 8 -iso-PG F2 $\alpha$ levels were also higher in hypertensive men than in normotensive men (HT: $2.99 \pm 0.26 \mathrm{nmol} / \mathrm{L}, \mathrm{n}=32$, NT: $1.96 \pm 0.21 \mathrm{nmol} / \mathrm{L}, \mathrm{n}=21, \mathrm{P}<0.01)$. In contrast, the plasma NO-metabolites were lower in hypertensive men than in normotensive men (HT: $34.5 \pm 2.2 \mu \mathrm{mol} / \mathrm{L}, \mathrm{n}=$ 32, NT: $55.4 \pm 4.4 \mu \mathrm{mol} / \mathrm{L}, \mathrm{n}=21, \mathrm{P}<0.001)$.

In the overall analysis of hypertensive and normotensive men, plasma total homocystine levels were correlated with plasma 8-iso-PG F2 $\alpha(\mathrm{r}=0.411, \mathrm{n}=53, \mathrm{P}<$ 0.01) (Figure 2). In addition, plasma NO-metabolites levels were inversely correlated with and plasma total homocystine $(\mathrm{r}=-0.315, \mathrm{n}=53, \mathrm{P}<0.05)$ (Figure 3), and plasma 8-iso-PG F2 $\alpha(\mathrm{r}=-0.300, \mathrm{n}=53, \mathrm{P}<0.05)$, respectively.

\subsection{Relationship between Membrane Fluidity of Red Blood Cells and Plasma Total Homocysteine, Plasma 8-Iso-Prostaglandin F2 $\alpha$, or Nitric Oxide-Metabolite Levels in Hypertensive and Normotensive Men}

The order parameter (S) of RBC membranes in the ESR spectra was correlated with plasma total homocysteine $(\mathrm{r}$ $=0.286, \mathrm{n}=53, \mathrm{P}<0.05)$ (Figure 4) and plasma 8 -iso-PG F2 $\alpha(\mathrm{r}=0.317, \mathrm{n}=53, \mathrm{P}<0.05)$, and inversely correlated with plasma NO-metabolites $(\mathrm{r}=-0.342, \mathrm{n}=$ 
53, $\mathrm{P}<0.05)$, suggesting that reduced membrane fluidity of RBCs might be associated with hyperhomocysteinemia, increased oxidative stress and endothelial dysfunction.

Multivariate regression analysis also showed that, after adjusting for general risk factors, plasma total homocysteine still remained as a significant determinant of membrane fluidity of RBCs (Table 2: Model 1). It was also demonstrated that plasma 8-iso-PG F2 $\alpha$ was an independent predictor of membrane fluidity of RBCs after adjusting for general risk factors (Table 3: Model 2).

\section{Discussion}

There is evidence showing that both hyperhomocysteinemia and oxidative stress might actively participate in the pathophysiology of hypertension, atherosclerosis, and other cardiovascular disease conditions [1-14], although associations of hyperhomocysteinemia and oxidative stress and their role in the regulation of membrane fluidity in hypertension remain to be solved. The present study was performed to evaluate the possible relationships among plasma total homocysteine, plasma 8-isoPG F2 $\alpha$ and membrane fluidity of RBCs in hypertensive

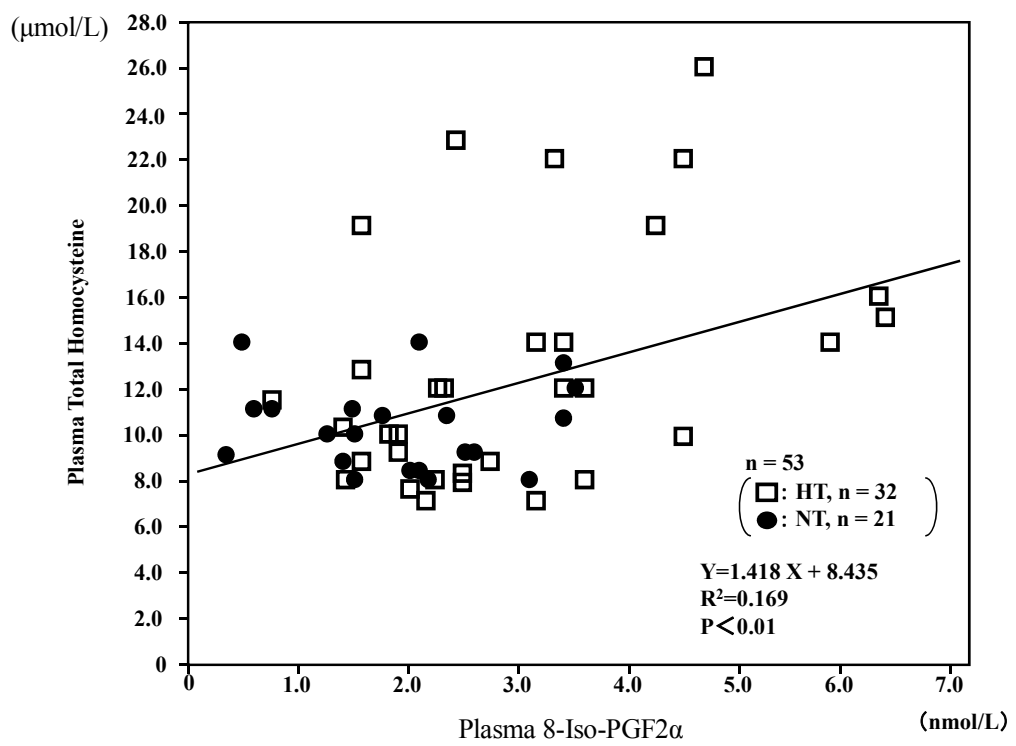

Figure 2. Correlation between plasma 8-iso-prostaglandin F2 $\alpha$ (8-iso-PG F2 $\alpha$ ) and plasma total homocysteine levels in hypertensive (HT) and normotensive (NT) men.

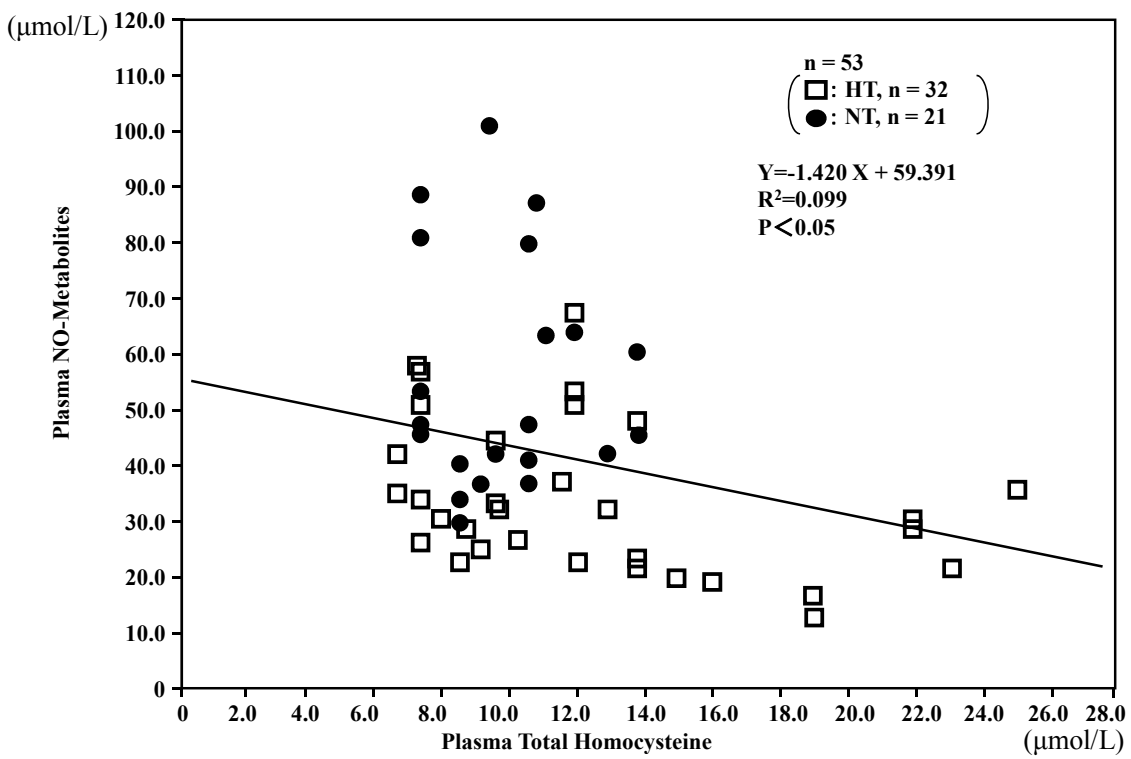

Figure 3. Inverse correlation between plasma total homocysteine levels and plasma nitric oxide (NO)-metabolite levels in hypertensive (HT) and normotensive (NT) men. 


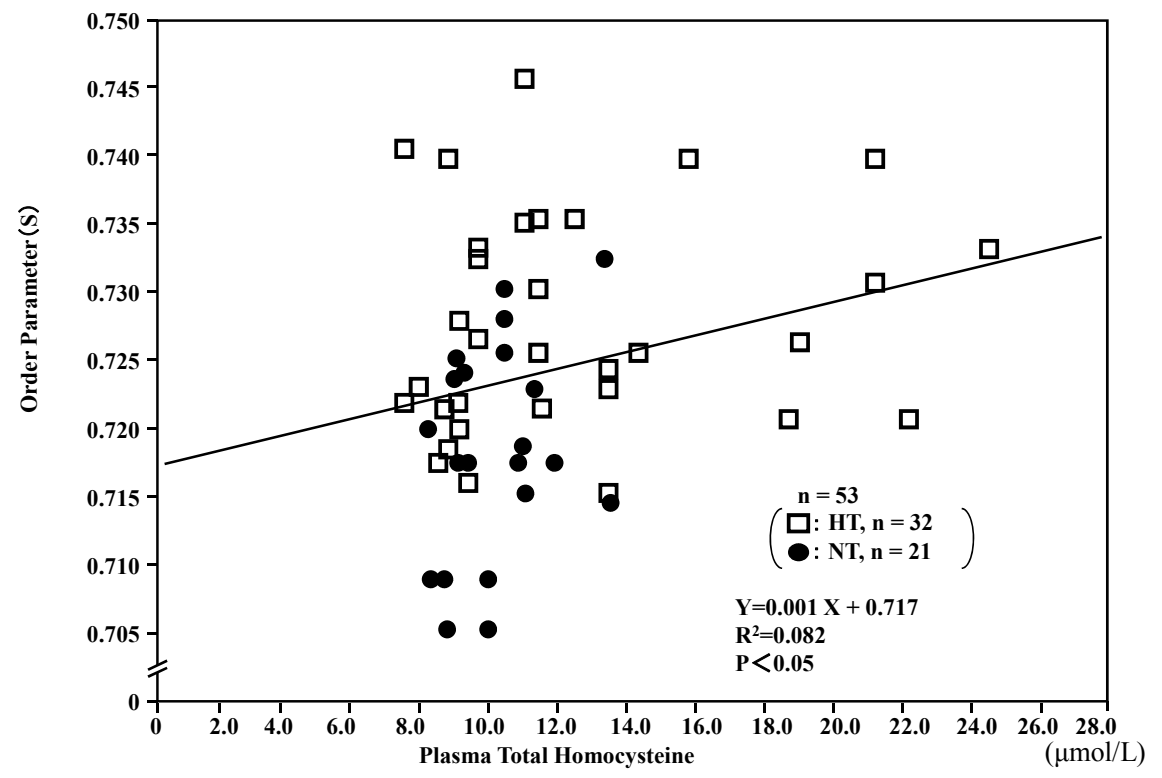

Figure 4. Correlation between plasma total homocysteine levels and membrane fluidity (order parameter; S) of red blood cells (RBCs) in hypertensive (HT) and normotensive (NT) men.

Table 2. Model 1: Multivariate regression analysis of plasma total homocysteine for predicting order parameter (S) of RBCs.

\begin{tabular}{cccc}
\hline & SRC & t-value & P-value \\
\hline Age (y.0.) & 0.07 & 0.416 & 0.6794 \\
Body mass index $\left(\mathbf{k g} / \mathbf{m}^{2}\right)$ & 0.051 & 0.32 & 0.7506 \\
Total cholesterol $(\mathrm{mg} / \mathrm{dL})$ & -0.137 & -0.829 & 0.4114 \\
Fasting plasma glucose $(\mathrm{mg} / \mathrm{dL})$ & 0.243 & 1.71 & $\mathbf{0 . 0 9 4 0}$ \\
$\begin{array}{c}\text { Systolic blood pressure (mm Hg) } \\
\text { Plasma total homocysteine } \\
(\mu \mathrm{mol} / \mathrm{L})\end{array}$ & 0.33 & $\mathbf{2 . 4 2 3}$ & $\mathbf{0 . 0 1 9 4}$ \\
\hline
\end{tabular}

$\overline{\mathrm{R}^{2}=0.254, \mathrm{n}=53, \mathrm{~F}=2.610, \mathrm{P}=0.0292 ; \text { SRC: } \text { standard regression coef- }}$ ficient

Table 3. Model 2: Multivariate regression analysis of plasma 8-iso-PGF2 $\alpha$ for predicting order parameter (S) of RBCs.

\begin{tabular}{cccc}
\hline & SRC & t-value & P-value \\
\hline Age (y.o.) & $6.295 \times 10^{-5}$ & $3.623 \times 10^{-4}$ & 0.9997 \\
Body mass index (kg/m²) & -0.088 & -0.532 & 0.5970 \\
Total cholesterol (mg/dL) & -0.218 & -1.247 & 0.2186 \\
$\begin{array}{c}\text { Fasting plasma glucose } \\
\text { (mg/dL) }\end{array}$ & 0.227 & 1.591 & 0.1184 \\
$\begin{array}{c}\text { Systolic blood pressure } \\
\text { (mm Hg) }\end{array}$ & 0.249 & 1.717 & 0.0927 \\
$\begin{array}{c}\text { Plasma 8-iso-PGF2 } \alpha \\
\text { (nmol/L) }\end{array}$ & 0.363 & 2.335 & 0.0240 \\
\hline
\end{tabular}

$\mathrm{R}^{2}=0.239, \mathrm{n}=53, \mathrm{~F}=2.413, \mathrm{P}=0.0413 ; \mathrm{RC}$ : standard regression coefficient and normotensive men using the ESR method. The results showed that the order parameter (S) of RBC membranes in the ESR spectra was significantly higher in hypertensive men than in normotensive men, indicating that membrane fluidity of RBCs was decreased in hypertension. The finding might be consistent with our previous findings showing that the cell membranes were stiffer and less fluid in hypertension [15,17-23].

In the present study, we showed that plasma total homocysteine and plasma 8-iso-PG F2 $\alpha$ (an index of oxidative stress) levels were significantly higher in hypertensive men than in normotensive men. In the overall analysis of hypertensive and normotensive men, plasma total homocystine levels were correlated with plasma 8-isoPG F2 $\alpha$ levels. Furthermore, it was shown that the order parameter $(\mathrm{S})$ of RBCs was correlated with plasma total homocyseine and plasma 8-iso-PG F2 $\alpha$ levels, indicating that reduced membrane fluidity of RBCs was associated with hyperhomocysteinemia and increased oxidative stress. Multivariate regression analysis also demonstrated that, after adjustment for general risk factors, plasma total homocysteine and plasma 8 -iso-PG F2 $\alpha$ were independent determinants of membrane fluidity of RBCs, respectively. Because the deformability of RBCs might be highly dependent on the membrane fluidity $[15,16]$, the reduction in membrane fluidity of RBCs could cause a disturbance in the blood rheologic behavior and the microcirculation in hypertension.

It was shown that shear rate, shear stress and blood viscosity were correlated with membrane fluidity of RBCs [24]. The finding proposed that in vivo shear forces 
might participate in the control of RBC membrane fluidity, and that RBCs might adapt their membrane properties to blood flow conditions. It was also demonstrated that $\mathrm{RBC}$ membranes might become more rigid after myocardial infarction, which could contribute to the decreased RBC deformability and the increased blood viscosity in this group of patients [25]. On the other hand, Cazzola et al. [26] reported that the membrane fluidity of RBCs was decreased in the obese subjects, and proposed that a decrease in RBC membrane fluidity could contribute to a reduction of the rate of blood flow and the oxygen diffusion through the RBC membranes and its exchange with tissues. It might be possible that alterations in RBC membrane fluidity with hyperhomocysteinemia and increased oxidative stress would be strongly linked to the progression of circulatory disorders.

The precise mechanisms by which hyperhomocysteinemia and oxidative stress could affect the membrane functions are not fully understood. Wang et al. [27] showed a significant association between serum homocysteine and oxidative stress in elderly patients with obstructive sleep apnea syndrome. It was also demonstrated that homocysteine increased the oxidative stress levels dose- and time-dependely in cultured bovine retinal endothelial cells [28]. These findings might be consistent with our present finding indicating that plasma total homocysteine levels were correlated with plasma 8-iso-PG F $2 \alpha$ levels. On the other hand, it was shown that endothelium-dependent vasodilation was impaired in subjects with hyperhomocysteinemia and elevated oxidative stress levels $[4,6,29,30]$. Stühlinger et al. [31] demonstrated that homocysteine might directly impair the NO synthase pathway in vitro. In a study presented earlier, we showed that an NO donor significantly improved membrane fluidity of RBCs in hypertensive subjects, suggesting that NO could have a beneficial effect on the rheologic behavior of RBCs and the microcirculation in hypertension [18]. In the present study, it was demonstrated that decreased plasma NO-metabolite levels were associated with not only reduced membrane fluidity of RBCs, but also hyperhomocysteinemia and increased 8-iso-PG F2 $\alpha$ levels. One hypothesis is that the effects of homocysteine and oxidative stress on membrane fluidity of RBCs might be mediated, at least in part, by reducing NO-bioavailability, although direct actions of homocysteine and oxidative stress on membrane structural and functional properties cannot be fully excluded [32,33]. Further studies should be performed to assess more precisely the interactions among homocysteine, oxidative stress and endothelial function, and their role in the regulation of membrane functions in hypertension.

\section{Conclusion}

The results of the present study demonstrated that both plasma total homocysteine and 8-iso-PG F2 $\alpha$ levels were elevated in hypertensive men compared with normotensive men. Furthermore, it was shown that reduced membrane fluidity of RBCs was correlated with increased plasma total homocysteine and 8-iso-PG F2 $\alpha$ levels, and decreased plasma NO metabolite levels, suggesting that abnormalities in RBC membranes in hypertension might be associated with hyperhomocysteinemia, increased oxidative stress, and endothelial dysfunction. Although this is a cross-sectional and correlative study in Japanese men, the results of the present study could provide a hypothesis that hyperhomocysteinemia and oxidative stress with endothelial dysfunction might have a close correlation with the impaired rheologic behavior of RBCs and the microcirculation, and contribute, at least in part, to the circulatory disorders in hypertensive men.

\section{Acknowledgements}

This study was supported in part by grants-in-aid for scientific research from the Ministry of Education, Science, Sports, Culture and Technology of Japan (15590604, 18590658, 20590710, 23590901), the Uehara Memorial Foundation (2005), the Takeda Science Foundation (2006), the Salt Science Foundation (2007), and the Mitsui Foundation (2008).

\section{REFERENCES}

[1] J. Danesh and S. Lewington, "Plasma Homocysteine and Coronary Heart Disease: Systemic Review of Published Epidemiological Studies," Journal of Cardiovascular Risk, Vol. 5, No. 4, 1998, pp. 229-232. doi: $10.1177 / 174182679800500404$

[2] D. S. Wald, M. Law and J. K. Morris, "Homocysteine and Cardiovascular Disease: Evidence on Causality from a Meta-Analysis," British Medical Journal, Vol. 325, No. 7374, 2002, pp. 1202-1206.

doi:10.1136/bmj.325.7374.1202

[3] L. J. Dominguez, A. Galioto, A. Pineo, A. Ferlisi, M. Ciaccio, E. Putignano, M. Belvedere, G. Costanza and M. Barbagallo, "Age, Homocysteine, and Oxidative Stress: Relation to Hypertension and Type 2 Diabetes Mellitus," Journal of the American College of Nutrition, Vol. 29, No. 1, 2010, pp. 1-6.

[4] A. Tawakol, T. Omland, M. Gerhard, J. T. Wu and M. A. Creager, "Hyperhomocysteinemia is Associated with Impaired Endothelium-Dependent Vasodilation in Humans," Circulation, Vol. 95, No. 5, 1997, pp. 1119-1121. doi:10.1161/01.CIR.95.5.1119

[5] D. D. Heistad, Y. Wakisaka, J. Miller, Y. Chu and R. Pena-Silva, "Novel Aspects of Oxidative Stress in Cardiovascular Diseases," Circulation Journal, Vol. 73, No. 2, 2009, pp. 201-207. doi:10.1253/circj.CJ-08-1082

[6] E. Schulz, T. Gori and T. Munzel, "Oxidative Stress and Endothelial Dysfunction in Hypertension," Hypertension 
Reseach, Vol. 34, No. 6, 2011, pp. 665-673. doi:10.1038/hr.2011.39

[7] A. Whaley-Connell, P. A. McCullough and J. R. Sowers, "The Oxidative Stress in the Metabolic Syndrome," Review in Cardiovascular Medicine, Vol. 12, No. 1, 2011, pp. 21-29.

[8] Q. Pu, M. F. Neves, A. Virdis, R. M. Touyz and E. L. Schiffrin, "Endothelin Antagonism on Aldosterone-Induced Oxidative Stress and Vascular Remodeling," $\mathrm{Hy}$ pertension, Vol. 42, No. 1, 2003, pp. 49-55. doi:10.1161/01.HYP.0000078357.92682.EC

[9] A. Virdis, E. Duranti and S. Taddei, "Oxidative Stress and Vascular Damage in Hypertension: Role of Angiotensin II," International Journal of Hypertension, Vol. 2011, 2011, Article ID: 916310. doi:10.406/2011/916310

[10] V. Dhawan and S. Jain, "Effect of Garlic Supplementation on Oxidized Low Density Lipoprotein and Lipid Peroxidation in Patients of Essential Hypertension," Molecular and Cellular Biochemistry, Vol. 266, No. 1-2, 2004, pp. 109-115. doi:10.1023/B:MCBI.0000049146.89059.53

[11] R. Rodrigo, H. Prat, W. Passalacqua, J. Araya, C. Guichard and J. P. Bachler, "Relationship between Oxidative Stress and Essential Hypertension," Hypertension Research, Vol. 30, No. 12, 2007, pp. 1159-1167. doi:10.1291/hypres.30.1159

[12] C. Vassalle, N. Botto, M. G. Andreassi, S. Berti and A. Biagini, "Evidence for Enhanced 8-Isoprostane Plasma Levels, as Index of Oxidative Stress in Vivo, in Patients with Coronary Artery Disease," Coronary Artery Disease, Vol. 14, No. 3, 2003, pp. 213-218. doi:10.1291/hypres.30.1159

[13] B. Wang, J. Pan, L. Wang, H. Zhu, R. Yu and Y. Zou, "Association of Plasma 8-Isoprostane Levels with the Presence and Extent of Coronary Stenosis in Patients with Coronary Artery Disease," Atherosclerosis, Vol. 184, No. 2, 2006, pp. 425-430.

[14] K. Tsuda, "Oxidative Stress and Membrane Fluidity of Red Blood Cells in Hypertensive and Normotensive Men. An Electron Spin Resonance Investigation," International Heart Journal, Vol. 51, No. 2, 2010, pp. 121-124. doi:10.1536/ihj.51.121

[15] J. Zicha, J. Kunes and M. A. Devynck, "Abnormalities of Membrane Function and Lipid Metabolism in Hypertension," American Journal of Hypertension, Vol. 12, No. 3, 1999, pp. 315-331.

[16] K. Tsuda and I. Nishio, "Membrane Fluidity and Hypertension," American Journal of Hypertension, Vol. 16, No. 3, 2003, pp. 259-261. doi:10.1016/S0895-7061(02)03257-0

[17] K. Tsuda, "Hyperhomocysteinemia and Membrane Fluidity of Red Blood Cells in Normotensive and Hypertensive Men: An Electron Spin Resonance Investigation," Clinical and Experimental Pharmacology and Physiology, Vol. 34, No. Supplement 1, 2007, pp. S70-S72. doi:10.1111/j.1440-1681.2007.04784.x

[18] K. Tsuda, K. Kimura, I. Nishio and Y. Masuyama, "Nitric Oxide Improves Membrane Fluidity of Erythrocytes in
Essential Hypertension-An Electron Paramagnetic Resonance Investigation," Biochemical and Biophysical Research Communications, Vol. 275, No. 3, 2000, pp. 946954. doi:10.1006/bbrc.2000.3408

[19] K. Tsuda, Y. Kinoshita, K. Kimura, I. Nishio and Y. Masuyama, "Electron Paramagnetic Resonance Investigation on Modulatory Effect of $17 \beta$-Estradiol on Membrane Fluidity of Erythrocytes in Postmenopausal Women," Arteriosclerosis, Thrombosis and Vascular Biology, Vol. 21, No. 8, 2001, pp. 1306-1312. doi:10.1161/hq0801.093507

[20] K. Tsuda, H. Iwahashi, Y. Minatogawa, I. Nishio, R. Kido and Y. Masuyama, "Electron Spin Resonance Studies of Erythrocytes from Spontaneously Hypertensive Rats and Humans with Essential Hypertension," Hypertension, Vol. 9, No. 6, Supplement III, 1987, pp. III19III24.

[21] K. Tsuda, Y. Kinoshita-Shimamoto, K. Kimura and I. Nishio, "Nitric Oxide Is a Determinant of Membrane Fluidity of Erythrocytes in Postmenopausal Women: An Electron Paramagnetic Resonance Investigation," American Journal of Hypertension, Vol. 16, No. 3, 2003, pp. 244-248. doi:10.1016/S0895-7061(02)03261-2

[22] K. Tsuda, "Role of Adiponectin and Oxidative Stress in the Regulation of Membrane Microviscosity of Red Blood Cells in Hypertensive and Normotensive Men: An Electron Spin Resonance Study," Journal of Obesity, Vol. 2011, 2011, Article ID: 548140. doi: $10.1155 / 2011 / 548140$

[23] K. Tsuda, "Associations of Oxidative Stress and Inflammation and Their Role in the Regulation of Membrane Fluidity of Red Blood Cells in Hypertensive and Normotensive Men: An Electron Spin Resonance Study," Advances in Bioscience and Biotechnology, Vol. 3, No. 7A, 2012, pp. 1020-1027. doi:10.4236/abb.2012.327124

[24] K. H. Le Sang Quan, J. Levenson, M. Del Pino, A. Simon and M. A. Devynck, "In Vivo Shear Flow and Erythrocyte Membrane Fluidity in Hypertensive Patients," British Journal of Clinical Pharmacology, Vol. 36, No. 5, 1993, pp. 437-443. doi:10.1111/j.1365-2125.1993.tb00392.x

[25] C. Saldanha, L. Sargento, J. Monteiro, C. Perdigao, C. Ribeiro and J. Martins-Silva, "Impairement of the Erythrocyte Membrane Fluidity in Survivors of Acute Myocardial Infarction. A Prospective Study," Clinical Hemorheology and Microcirculation, Vol. 20, No. 2, 1999, pp. 111-116.

[26] R. Cazzola, M. Rondanelli, S. Russo-Volpe, E. Ferrari and B. Cestaro, "Decreased Membrane Fluidity and Altered Susceptibility to Peroxidation and Lipid Composition in Overweight and Obese Female Erythrocytes," Journal of Lipid Research, Vol. 45, No. 10, 2004, pp. 1846-1851. doi:10.1194/jlr.M300509-JLR200

[27] L. Wang, J. Li, Y. Xie and X. G. Zhang, "Association between Serum Homocysteine and Oxidative Stress in Elderly Patients with Obstructive Sleep Apnea/Hypopnea Syndrome," Biomedical and Environmental Sciences, Vol. 23, No. 1, 2010, pp. 42-47. doi:10.1016/S0895-3988(10)60030-X

[28] S. R. Bharathi Devi, G. Suganeswari, T. Sharma, M. Thennarasu and N. Angayarkanni, "Homocysteine In- 
Fluidity of Red Blood Cells in Hypertensive and Normotensive Men: An Electron Spin Resonance Investigation

duces Oxidative Stress in Young Adult Central Retinal Vein Occlusion," British Journal of Ophthalmology, Vol. 96, No. 8, 2012, pp. 1122-1126.

doi:10.1136/bjophthalmol-2011-301370

[29] L. Loffredo, A. Marcoccia, P. Pignatelli, P. Andreozzi, M. C. Borgia, R. Cangemi, F. Chiarotti and F. Violi, "Oxidative-Stress-Mediated Arterial Dysfunction in Patients with Peripheral Arterial Disease," European Heart Journal, Vol. 28, No. 5, 2007, pp. 608-612. doi:10.1093/eurheartj/ehl533

[30] M. Annuk, M. Zilmer, L. Lind, T. Linde and B. Fellstrom, "Oxidative Stress and Endothelial Function in Chronic Renal Failure," Journal of American Society of Nephrology, Vol. 12, No. 12, 2001, pp. 2747-2752.

[31] M. C. Stühlinger, P. S. Tsao, J. H. Her, M. Kimoto, R. F.
Balint and J. P. Cooke, "Homocysteine Impairs the Nitric Oxide Synthase Pathway. Role of Asymmetric Dimethylarginine," Circulation, Vol. 104, No. 21, 2001, pp. 25692575. doi:10.1161/hc4601.098514

[32] C. G. Zou, N. S. Agar and G. L. Jones, "Oxidative Insult to Human Red Blood Cells Induced by Free Radical Initiator AAPH and Its Inhibition by Commercial Antioxidant Mixture," Life Science, Vol. 69, No. 1, 2001, pp. 7586. doi:10.1016/S0024-3205(01)01112-2

[33] F. Lenfant, J. J. Lahet, C. Vergely, F. Volot, M. Freysz and M. Rochette, "Lidocaine Inhibits Potassium Efflux and Hemolysis in Erythrocytes During Oxidative Stress in Vitro," General Pharmacology, Vol. 34, No. 3, 2000, pp. 193-199. 